\title{
A case of sarcoma of the central nervous system presenting as a Guillain-Barré syndrome
}

\author{
N BISHOP, A CHAKRABARTI, D PIERCY, DGF HARRIMAN*, JMS PEARCE \\ From the Hull Royal Infirmary, Hull, and the Department of Neuropathology*, Leeds General Infirmary, \\ Leeds, $U K$
}

SUMMARY A case of widespread spinal sarcoma is described with meningeal infiltration, involvement of the anterior cranial fossa complicated by aplastic anaemia and visceral metastases.

Sarcomas of the central nervous system are extremely rare and are generally of intracranial origin. The clinical features are usually those of a space occupying or destructive lesion within the CNS. The Guillain-Barré syndrome has been associated with malignancy, though no cases appeared to have direct tumour involvement of the spinal roots. ${ }^{1}$ The case we describe is thus unusual for its clinical presentation, the apparent intraspinal origin of the tumour and the associated visceral metastases.

\section{Case report}

The patient, a 27-year-old farmer presented with a 9 week history of progressive weakness of the legs, the left being worse than the right. There were no sensory symptoms or disturbances of autonomic function, although he did complain of left sided headaches during the preceding 2 weeks. Seven weeks previously he had developed "viral" hepatitis with cholestasis. Apart from a 7 year history of periodic low back pain, there were no other significant details in his past medical or family history. On examination at that time he was noted to have bilateral papilloedema (with normal visual acuity). The other cranial nerves were normal. Neurologically the arms were normal but there was marked wasting and weakness of the proximal muscles in the lower limbs, and some weakness of dorsiflexion of the left foot. Knee and ankle jerks were absent bilaterally and plantar responses were flexor. There was no sensory impairment in the legs. One patch of freckling was noted in the left axilla, otherwise systemic examination was normal. CSF examination revealed clear fluid at a pressure of 32 $\mathrm{cm}$ of CSF with a white cell count of 9 lymphocytes $/ \mathrm{cmm}$

Address for reprint requests: Dr J Pearce, Dept of Neurology, Hull Royal Infirmary, Hull, HU3 2JZ, UK.

Received 27 August 1982 and in revised form 28 October 1982. Accepted 20 November 1982 and a protein of $1.86 \mathrm{~g} / \mathrm{l}$. CT scan showed minimal dilatation of the lateral ventricles but no evidence of a space occupying lesion. A cervical and lumbar myelogram was normal apart from a small filling defect on the left side in one of the root pouches at the L3 level.

Clinically the most likely diagnosis was of a GuillainBarré syndrome with the papilloedema possibly resulting from CSF malabsorption due to the high protein content. ${ }^{2}$ Whilst in hospital his weakness improved, but four weeks after discharge he developed herpes zoster in a T9 distribution on the right side. Two months later he had a 30 minute transient episode of sudden dysphasia with numbness in the right hand. His visual acuity had fallen to $\mathrm{J} 4$ and the mild bilateral papilloedema persisted. A repeat CT scan showed a parapituitary tumour indenting the third ventricle causing internal hydrocephalus. Following angiography and ventriculography the lesion was explored. At operation a mass was found both in the region of the lamina terminalis and the posterior part of the left gyrus rectus extending into the hippocampal gyrus. A biopsy was taken of this latter part of what was presumed to be the same mass. The histology showed no tumour cells, only gliosis.

The patient was treated initially with radiotherapy and steroids, with a presumptive diagnosis of a glioma of the floor of the third ventricle.

Five months later he developed aplastic anaemia for which no cause was found. Haemoglobin $7.5 \mathrm{~g} / \mathrm{dl}$; pancytopenia on blood film examination with a white cell count of $0.8 \times 10^{9} / \mathrm{l}$ and platelet count of $40000 \times 10^{9} / 1$. Bone marrow examination-severe hypoplasia. This was managed with frequent blood transfusions and oxymethalone $50 \mathrm{mg}$ four times a day. Two months later over a period of five days he developed an increasing quadriparesis, with bilateral extensor plantar responses and a sensory level at C5. A myelogram showed a complete block at $\mathrm{C} 4$ with multiple defects in the cervical region. A decompression laminectomy was performed. At operation multiple intra-dural, extramedullary deposits of tumour were seen, and the large nodule at the $C 4$ level excised.

Histology of the operative specimen showed round, polyhedral and spindle shaped cells; the nuclei tended to be vesicular and contained prominent nucleoli. It was con- 


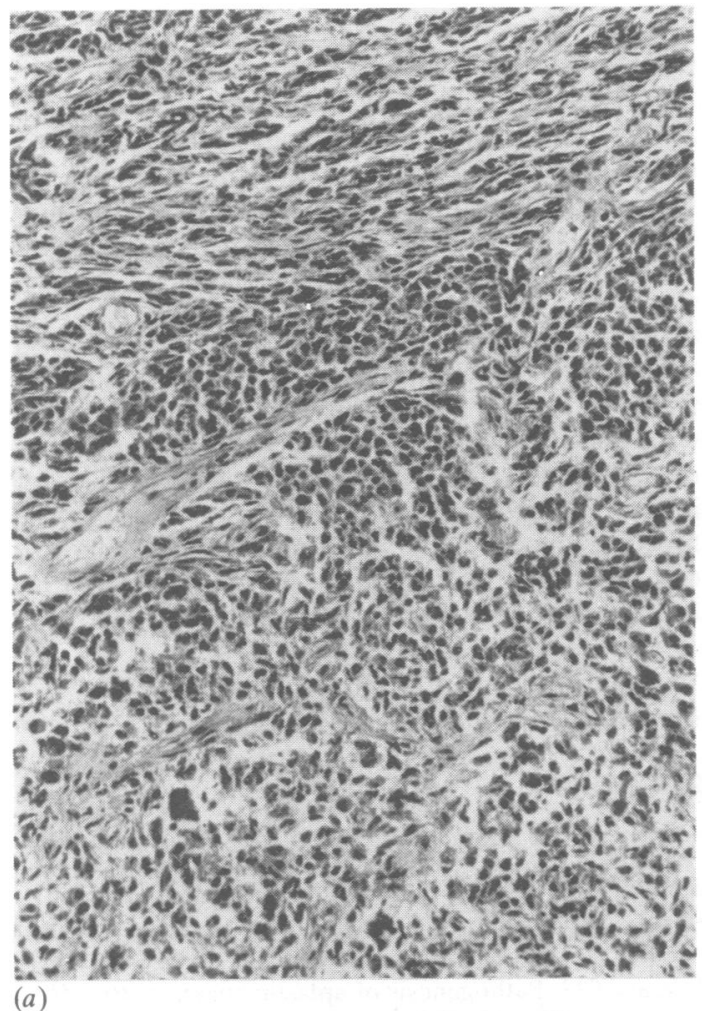

(a)

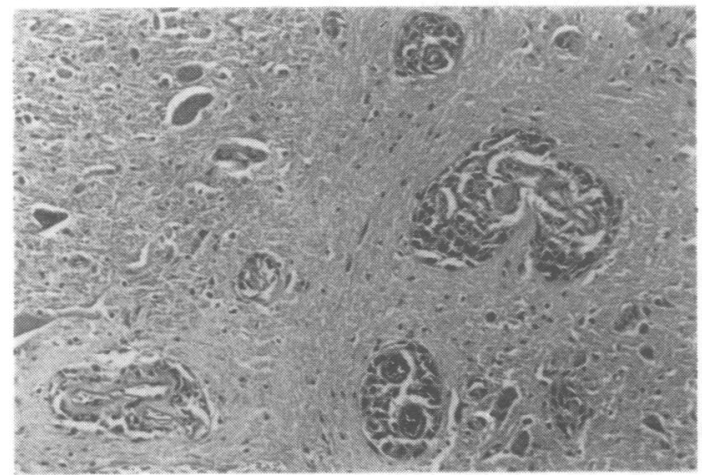

(c)

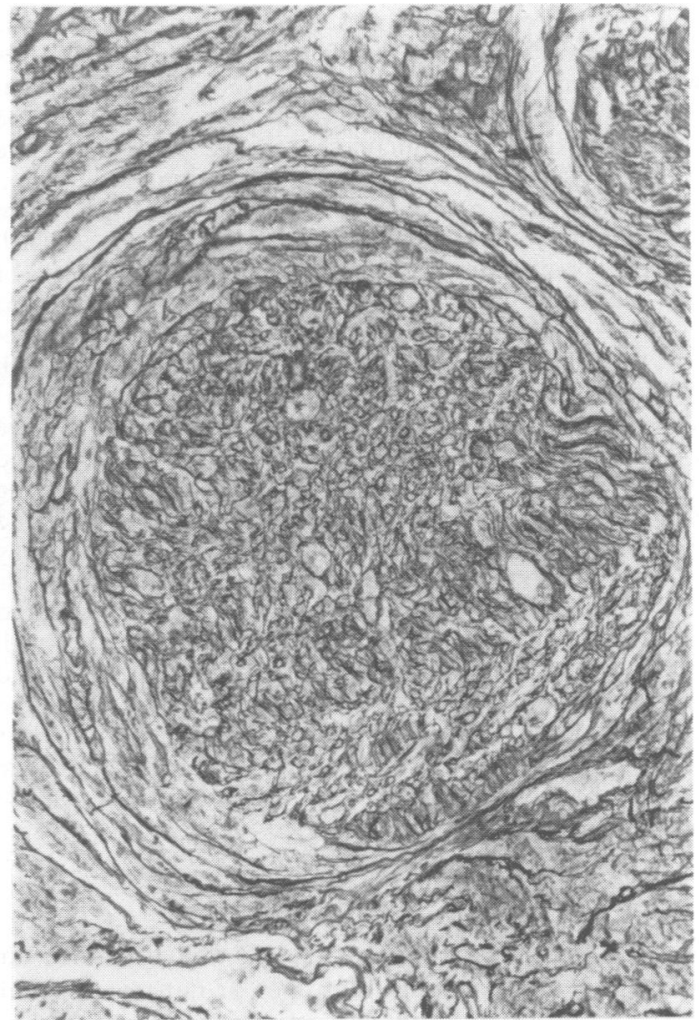

(b)

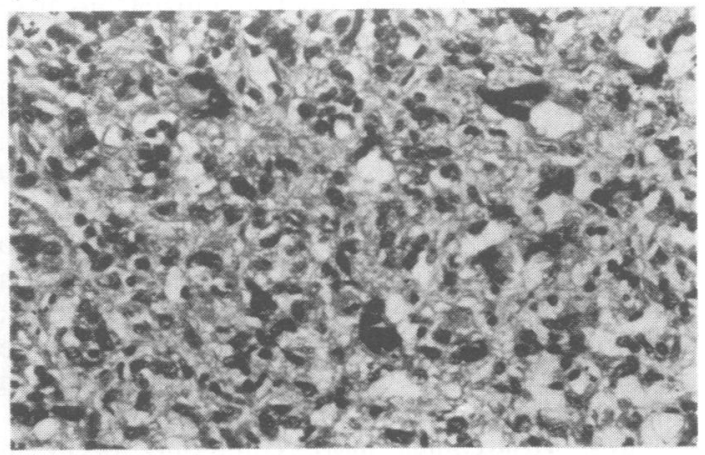

(d)

Fig 1(a) Tumour in spinal meninges ( $H \& E \times 190$ ). To show spindle cell and pleomorphic cell areas. (b) Reticulin staining of infiltrated spinal root $(\times 190)$. The reticulin framework remains undisturbed despite tumour inflitration. (c) Perivascular infiltration by tumour cells within the spinal cord $(H \& E \times 480)$. (d) Cerebral meninges containing highly pleomorphic tumour cells $(H \& E \times 480)$.

sidered to be a malignant neoplasm of undifferentiated type adherent to the meninges.

The power in the patient's limbs improved following the decompression, but over the next month his general condition declined and he died. There had been no remission of the aplastic anaemia.
Post-mortem examination revealed a midline intracranial mass on the floor of the anterior fossa anterior to the pituitary fossa. The spinal cord showed the presence of two intradural tumourous masses on its anterior aspect at the level of C5 vertebra. The pleural surface of the right lung was covered by irregular small firm plaques. The right hilar 
and paratracheal lymph nodes were enlarged by metastatic tumour tissue. In the liver small white subcapsular nodules were present in both lobes. There were also several irregular nodules of metastatic tumour within the spleen. The remainder of the examination was unremarkable, there being no evidence of neurofibromatosis or tumour invasion of the bone marrow. Microscopically, some of the spinal cord tumour was leptomeningeal, with two cell types which tended to merge in some areas. The first type consisted of narrow spindle cells in wide streams in places accompanied by bands of collagen (the upper part of fig 1(a)). The second type (fig 1(a), lower) consisted of masses of cells with pleomorphic nuclei, occasionally multinucleated and variable amounts of cytoplasm. Many of the spinal roots were encircled or infiltrated by tumour, of the spindle celled type. The reticulin in these roots was little disturbed (fig 1(b)). The substance of the spinal cord at one level showed perivascular infiltration by the tumour, (fig 1(c)). Histology of the brain showed extensive meningeal spread of undifferentiated tumour tissue (fig 1(d)). The tumour entered the midbrain to join perivascular mantles as in the cord. Four particularly large masses of both spindle celled and sarcomatous growth were found on the skull base, in the anterior fossa overlying an air sinus. Histology of the hilar lymph nodes and spleen confirmed metastatic spread of the tumour. The bone marrow microscopically remained free from tumour.

\section{Discussion}

The clinical presentation suggests that the tumour originated in the spinal meninges or nerves and that the papilloedema and subsequent cerebral symptoms were due to intracranial meningeal involvement and the parasellar tumour. The aetiology of the aplastic anaemia remains obscure. Two possible explanations are infectious hepatitis and a course of sulphonamides used to cover his ventriculography. However, the delay between these events was 6-9 months rather than the expected $10-12$ weeks. ${ }^{34}$

It is difficult to give a precise histological label to the type of sarcoma described in this case although there are three possibilities:- neurofibrosarcoma, fibrosarcoma, or meningeal sarcoma. All of these malignant tumours arise in pre-existing benign tumours, which makes identification much easier. ${ }^{5}$

Neurofibrosarcomas more commonly occur in patients with neurofibromatosis. ${ }^{611}$ In this case there were no pre-existing benign tumours and a patch of freckling in the left axilla was the only possible cutaneous manifestation of neurofibromatosis. Thus the diagnosis of neurofibrosarcoma seems improbable. Most intra-cranial sarcomas are of meningeal origin $^{7}$ and primary sarcomas of the meninges have been described though these generally occur in infants and children with a rapid downhill course. ${ }^{8}$ Primary intra-spinal sarcomas of the same origin must be very rare. Reviews of intracranial sarcomas $^{910}$ emphasise their poor prognosis particularly with the polymorphocellular and spindle celled types of tumour. Sub-arachnoid spread may occur with such tumours though visceral deposits have only been described in two patients. ${ }^{89}$ The histology in our patient can go no further than suggesting fibrosarcoma or meningeal sarcoma. The nature of the growth within the spinal nerve roots is slightly in favour of the former.

We thank Mr MR Gooding for neurosurgical assistance and Mrs YM Garner for secretarial services.

\section{References}

${ }^{1}$ Kennedy RH, Danielson MA, Mulder DW, Kurland LT. Guillain-Barré syndrome-a 42 year epidemiologic and clinical study. Mayo Clin Proc 1978;53:93-9.

${ }^{2}$ Brain's Diseases of the Nervous System, 8th ed. Walton JN, ed London: Oxford University Press. 1977:951.

${ }^{3}$ Geary CG. Pathogenesis of aplastic anaemia. Br J Hosp Med 1979;392-402.

${ }^{4}$ Clinics in Haematology October 1980. Gordon-Smith EC, ed London: WB Saunders. Vol 9 No. 3:653.

${ }^{5}$ Asbury AK, Johnson PC. Pathology of the Peripheral Nerve. Philadelphia: WB Saunders, 1978:224.

${ }^{6}$ Hope DG, Mulvihill JJ. Malignancy in neurofibromatosis. Adv Neurol 1981;29:40.

${ }^{7}$ Northfield DWC. The Surgery of the Central Nervous System. London:Blackwell, 1973:222-3.

${ }^{8}$ Russell DS, Rubinstein LJ. Pathology of Tumours of the Nervous System. 4th ed. London: Edward Arnold. 1977:92-3.

9 Christensen E, Lara DE. Intracranial sarcomas. $J$. Neuropath Exp Neurol 1953;12:41-56.

${ }^{10}$ Kernohan JW. Uihlein A. Sarcomas of the Brain. Springfield: Thomas, 1962.

"Pearce JMS. The central nervous system pathology in multiple neurofibromatosis. Neurology (Minneap) 1967;17:691-7. 